\title{
Mild clinical presentation in KLHL40-related Nemaline Myopathy (NEM 8)
}

Andreea M. Seferian ${ }^{a}$, Edoardo Malfatti b,c,d ${ }^{b}$ Caroline Bosson ${ }^{e}$, Laurent Pelletier e, Jessica Taytard f, Veronique Forin 9, Teresa Gidaro a, Elena Gargaun a, Pierre Carlier $^{\mathrm{h}}$, Julien Fauré e, Norma B. Romero ${ }^{\mathrm{b}, \mathrm{c}, \mathrm{d}}$, John Rendu ${ }^{\mathrm{e}}$, Laurent Servais ${ }^{\mathrm{a}}$

a I-Motion - Research center for pediatric neuromuscular diseases, Armand Trousseau Hospital, Paris, France

b Sorbonne Universities, UPMC Univ Paris 06, INSERM UMRS974, CNRS FRE3617, Center for Research in Myology, GHU La Pitié-Salpêtrière, Paris, France

c Unit of neuromuscular morphology, Institute of Myology, GHU La Pitié-Salpêtrière; Paris, France.

${ }^{d}$ Reference center for neuromuscular pathologies for Eastern Paris, Institute of Myology, AP-HP, GHU La Pitié-Salpêtrière, Paris, France

${ }^{\text {e }}$ Departement of biochemistry, molecular biochemistry and genetics, toxicology and pharmacology, Grenoble Alpes University, GIN Institute of Neurosciences, Grenoble, France

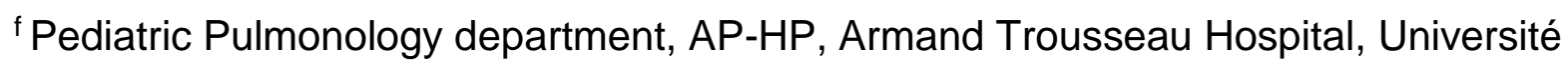
Pierre et Marie Curie-Paris6, Inserm U938, Paris, France

g Pediatric Rehabilitation Department, AP-HP, Armand Trousseau Hospital, Paris, France

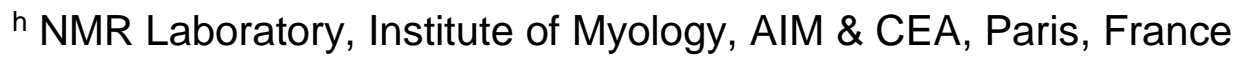


${ }^{*}$ Corresponding author: Andreea Seferian, MD \& Laurent Servais, MD, PhD, I-Motion

- Plateforme d'essais cliniques pédiatriques, Hôpital Armand Trousseau, Bâtiment

Lemariey, Porte 20, 2ème étage 26 Avenue du Dr Arnold Netter 75012 Paris, France.

I.servais@institut-myologie.org

a.seferian@institut-myologie.org

Tél : + $33(0) 171738050$

Fax : + $33(0) 144736583$ 


\section{Abstract}

Nemaline myopathies (NM) are clinically and genetically heterogeneous muscle diseases characterized by the presence of nemaline bodies (rods) in muscle fibers. Mutations in the $K L H L 40$ (kelch-like family member 40) gene (NEM 8) are common cause of severe/lethal nemaline myopathy. We report on an 8-year-old girl born to consanguineous Moroccan parents, who presented with hypotonia and poor sucking at birth, delayed motor development, and further mild difficulties in walking and fatigability. A muscle biopsy revealed the presence of nemaline bodies. $K L H L 40$ gene Sanger sequencing disclosed a never reported pathogenic homozygous mutation who resulted in absent KLHL40 protein expression in muscle. This further expand the phenotypical spectrum of KLHL40 related nemaline myopathy.

Keywords: Nemaline Myopathy; KLHL40 


\section{Introduction}

Nemaline myopathy (NM) is a rare congenital muscle disorder associating hypotonia, muscle weakness, and often skeletal deformities with the presence of nemaline bodies (rods) in muscle fibers [1]. This disorder has a marked clinical variability, ranging from neonatal lethal to mild non-progressive forms with onset in childhood and adulthood. NM has been classified into six clinical categories according to the severity of the disease, the age of onset and the pattern of muscle weakness [2].

To date, eleven genes have been identified for NM: a-skeletal actin (ACTA1, MIM\#161800) [3], muscle specific cofilin (CFL2, MIM\#610687) [4], nebulin (NEB, MIM\#256030) [5], slow troponin T (TNNT1, MIM\#605355) [6], $\beta$-tropomyosin (TPM2, MIM\#609285) [7], slow a-trompomyosine (TPM3, MIM\#609284) [8], kelch-repeat and BTB-[POZ]-domain containing 13) (KBTBD13, MIM\#609273) [9] and kelch-like family member 40 (KLHL40, MIM\#615340) [10] and member 41 (KLHL41, MIM\#607701) [11], leiomodin 3 (LMOD3, MIM\#616112) [12] and very recently myosin XVIIIB (MYO18B) [13].

Mutations in the KLHL4O gene (NEM 8) have been associated with severe/lethal NM [10]. KLHL40 belongs to the superfamily of kelch-repeat-containing proteins [14] and is crucial for myogenesis through regulation of E2F1-DP1 [15] and for skeletal muscle maintenance, binding to NEB and LMOD3 .

Almost all (96\%) of 32 affected individuals described in the seminal paper by

Ravenscroft et al. had acute respiratory failure, swallowing problems. Foetal akinesia, hypokinesia and contractures were present at birth in more the three quarters of the cases. The average age at death was 5 months $(n=14)$ [10]. Later on, Kawase et al. described a $K L H L 40$ mutated patient with congenital locked-in state, who died at 4 years [17]. More recently, mutations in KLHL4O have been described in a 9-year old 
female with severe NM patient and myastenic features, who surprisingly responded to acetylcolinestherase inhibitors [18].

Here we describe a mild NM myopathy case presenting a novel KLHL4O homozygous mutation.

\section{Case Report}

We present the case of a 8 year old girl third born to Moroccan consanguineous (the parents were first degree cousins) parents with no familial history for neuromuscular conditions. Of note congenital cataract was found in one of her two older sisters. Pregnancy was uneventful and she was born at 39 gestational weeks with a weight of 3200 grams ( $25^{\text {th }}$ percentile), a length of $50 \mathrm{~cm}\left(50^{\text {th }}\right.$ percentile) and a cranial circumference of $39 \mathrm{~cm}\left(97^{\text {th }}\right.$ percentile). At three days of age she was admitted in a neonatal unit due to axial hypotonia and poor sucking. She was on nasogastric feeding until the $15^{\text {th }}$ day, after which she was released on oral nutrition. Her evolution slowly improved. At 7 months, hypomimia and equinus deformity of the foot were noticed. The patient was able to support her head at 6 months. Walking was achieved at around 20 months.

At 3 years, she was able to run and ride a bike but had difficulties climbing the stairs. She had a waddling gait, deficit of the orbicularis oculi and drooling.

At five years, she had a good function of upper and lower limbs but all the activities were performed very slowly. The neck flexors and extensors were weak. At six years and a half, the Gowers sign was negative. The neck extensors were stronger than the flexors and inversely the knee extensors weaker than the flexors. From the respiratory point of view, multiple ear infections led to a mild hearing loss in the left 
ear. At 7 years, the patient was complaining a lot about tiredness and she was only able to walk for 10 minutes. She was using a manual wheel chair for long distances. She had predominant axial weakness. Coughing was rather inefficient and the sniff nasal inspiratory pressure was low.

Last seen at 8 years and 2 months, she weighed $20 \mathrm{~kg}\left(5^{\text {th }}\right.$ percentile), measured $129 \mathrm{~cm}$ (50th percentile), and was of normal intelligence (no formal IQ test was performed) (Fig. 1). She walked 599 meters at the 6-minute walking test. She was able to climb some steps without the rail. The Gowers sign was negative She had been under Tyrosine treatment $500 \mathrm{mg}$ b.i.d. for one year and a half, with mild and non-objective improvement; her mother found that she was doing "slightly better" with the treatment. A complete muscular testing was performed using the Medical Research Council 6-point score (MRC). Flexors and extensor of the hip, knee and foot, abductors and adductors of the hip on both sides were graded all at 4 ; with the exception of the right knee extensor (graded 5) and the hip adductors (graded 3) on both sides. The right upper limb strength was slightly higher for the elbow flexors, finger and wrist flexors and extensors (graded 5) ${ }^{\circ}$ compared to the left side (graded 4). The neck extensors were graded 3 compared to the flexors (graded 2).

During the first months of life, an intensive research of inborn errors of metabolism was performed (serum amino acid chromatography, carnitine, acyl-carnitine, lactic acid, vacuolated peripheral blood lymphocytes, CDG syndrome, urinary amino acid and organic acid chromatography) and no specific abnormality was detected. The cerebral neonatal MRI showed T2 hyper intensity of the periventricular white matter with nonspecific high diffusion. The karyotype was $X Y$ and methylation analysis by Southern blotting for Prader Willi syndrome and CTG sequence repeats analysis in DMPK gene by PCR and TP-PCR on the two strands for Steinert myotonic dystrophy 
were negative. The whole body $3 T$ MRI was performed at the age of 9 . It showed no particular changes of the muscles. The T2 cartography of the muscular tissue of the tights and calves showed normal values.

A deltoid muscle biopsy performed at 3 years of age showed the presence of clusters of elongated protein inclusions found both in cytoplasmic and subsarcolemmal areas of around $30 \%$ of fibres, staining red with the Gomori thricrome (Fig.2 A,B). The inclusions corresponded to nemaline bodies. Ultrastructural analysis confirmed the presence of nemaline bodies presenting the tipycal Z-disk electrondensity and net like organization. (Fig. 2 C,D).

NM gene panel Sanger sequencing of ACTA1, TPM2, TPM3, CFL2, TNNT1, KBTBD13, KLHL40, KLHL41 and $L M O D 3$ revealed a homozygous $K L H L 40$ gene c. $1498 \mathrm{C}>\mathrm{T}$ missense mutation leading to a p.Arg500Cys change at the protein level. The mutation was found only one time in the DIVAS database at an heterozygous level [19]. As the North African population was underrepresented in genetics databases, we screened an Arabic cohort present in the laboratory (100 individuals). The c.1498C>T transition was not found.

The p.Arg500Cys occurs in a conserved domain in the fourth Kelch Repeat (Fig 3 E) and is predicted to be deleterious according to prediction software (Polyphen,SIFT, CADD, Fathmm, PROVEAN) [20]. Based on KLHL40 PDB structure 4ASC, the Arg500 residue interacted with the Glu 528 inside a beta sheet by ionic interaction (Fig 3 B). The foldX software predicts an important variation of free energy [21]. 
The segregation was confirmed in both parents who carried the c.1498C $>\mathrm{T}$ at heterozygous state. One sister was heterozygous carrier of the mutation. (Fig $3 \mathrm{~A}$, C).

Western blot analysis performed using a rabbit polyclonal KLHL40 (KBTBD5) antibody from sigma (HPA024463) on protein extracted from skeletal muscle revealed absence of KLHL40 protein (Fig.2 E). RT-PCR analysis revealed that KLHL40 transcript was found overexpressed in the patient biopsy compared to a same age control biopsy (fig $3 \mathrm{D}$ ).

\section{Discussion}

We report the case of 8 year old girl with $K L H L 40$ gene-related nemaline myopathy, carrying a new mutation, c. $1498 \mathrm{C}>\mathrm{T}$. Her clinical phenotype is milder compared to those described in the literature.

Nemaline myopathy related to $K L H L 40$ varies very much in severity from neonatal death to very rare cases of survival into adolescence.

The most frequent mutation in patients with $K L H L 40$ NEM, c.1582G>A has been found in Japanese, Kurdish and Turkish persons. Ravenscroft et al. showed that c. $1582 \mathrm{G}>\mathrm{A}$ mutation suggests a milder phenotype compared to the cases where this mutation is absent. However, regardless of the genotype, the severity of the disease differs greatly, from death at 20 days to survival at adolescence. One explication could be given by modifying factors [10]. 
The screening of 129 probands with a milder NEM phenotype did not identify any $K L H L 40$ mutation, confirming that the mutations found by Ravenscroft et al. are probably restricted to severe cases [10].

Regarding the present mutation, it is noteworthy that it leads to increased transcript, but absent protein on the western blot. An explication could reside in a deep restructuration of the protein that expresses a new epitope not recognized by the antibody used in the Western blot. Another possibility is that the protein is either not produced, or it is rapidly degraded. We could assume that other proteins might possibly take over KLHL40 function to compensate for its loss but this is yet to be determined. Indeed, complete absence of the protein is otherwise difficult to conciliate with the milder phenotype

At the current time, there are insufficient MRI data to comment upon whether KLHL4O mutations give rise to specific patterns of muscle involvement, as for ACTA1 (proximal leg involvement) or NEB (predominant distal leg atrophy) [22].

A limitation of the present report is that it concerns a single case. However, KLHL4O is a recognized cause of nemaline myopathy and other nemaline-myopathy genes have been ruled out. The mutation is predicted to be pathogenic, as it is absent from the healthy population. Finally, western blot analysis revealed the absence of KLHL40 protein expression supporting the pathogenicity of c.1498C>T.

A recent report [18] demonstrated the dramatic effect of pyridostigmine in a patient with $K L H L 40$ severe phenotype. Pyridostigmine or other neuromuscular junctionacting molecules were not tried in the present case for several reasons: the clinical features of our patient (myopathic facies with no opthalmoplegia and lack of variability of muscular signs) did not determine us to start an anticholinergic 
treatment; another reason was that our patient was already on Tyrosine and we tried to avoid multiple therapy in order to assess Tyrosine efficacy.It is likely that other missense mutations in the $K L H L 40$ will be associated with such milder phenotype in the coming years. Phenotype-genotype relation are important to recognize, not only to avoid diagnosis misleading, but also to adequately inform the parents at the time of diagnosis, or in case of prenatal counselling.

Acknowledgements: The authors would like to thank G Brochier, A Madelaine, M Beuvin and E Lacène from 'Risler Laboratory (GHU La Pitié-Salpêtrière) for the electronic microscopy and Western blot studies respectively,P. Ray for the Arabic control cohort, and S. Gilabert for the muscular evaluation.

\section{References}

[1] North KN, Laing NG, Wallgren-Pettersson C. Nemaline myopathy: current concepts. The ENMC International Consortium and Nemaline Myopathy. J Med Genet 1997;34:705-13.

[2] Wallgren-Pettersson C, Laing NG. Report of the 70th ENMC International Workshop: nemaline myopathy, 11-13 June 1999, Naarden, The Netherlands. Neuromuscul Disord 2000;10:299-306.

[3] Nowak KJ, Wattanasirichaigoon D, Goebel HH, et al. Mutations in the skeletal muscle alpha-actin gene in patients with actin myopathy and nemaline myopathy. Nat Genet 1999;23:208-12. 
[4] Agrawal PB, Greenleaf RS, Tomczak KK, et al. Nemaline myopathy with minicores caused by mutation of the CFL2 gene encoding the skeletal muscle actinbinding protein, cofilin-2. Am J Hum Genet 2007;80:162-7.

[5] Lehtokari VL, Pelin K, Sandbacka M, et al. Identification of 45 novel mutations in the nebulin gene associated with autosomal recessive nemaline myopathy. Hum Mutat 2006;27:946-56.

[6] Johnston JJ, Kelley RI, Crawford TO, et al. A novel nemaline myopathy in the Amish caused by a mutation in troponin T1. Am J Hum Genet 2000;67:814-21.

[7] Donner K, Ollikainen M, Ridanpaa M, et al. Mutations in the beta-tropomyosin (TPM2) gene--a rare cause of nemaline myopathy. Neuromuscul Disord 2002;12:151-8.

[8] Laing NG, Wilton SD, Akkari PA, et al. A mutation in the alpha tropomyosin gene TPM3 associated with autosomal dominant nemaline myopathy NEM1. Nat Genet 1995;10:249.

[9] Sambuughin N, Swietnicki W, Techtmann S, et al. KBTBD13 interacts with Cullin 3 to form a functional ubiquitin ligase. Biochem Biophys Res Commun 2012;421:743-9.

[10] Ravenscroft G, Miyatake S, Lehtokari VL, et al. Mutations in KLHL40 are a frequent cause of severe autosomal-recessive nemaline myopathy. Am J Hum Genet 2013;93:6-18.

[11] Gupta VA, Ravenscroft G, Shaheen R, et al. Identification of KLHL41 Mutations Implicates BTB-Kelch-Mediated Ubiquitination as an Alternate Pathway to Myofibrillar Disruption in Nemaline Myopathy. Am J Hum Genet 2013;93:1108-17. 
[12] Yuen M, Sandaradura SA, Dowling JJ, et al. Leiomodin-3 dysfunction results in thin filament disorganization and nemaline myopathy. J Clin Invest 2015;125:4567.

[13] Malfatti E, Bohm J, Lacene E, Romero N, Laporte J. A premature stop codon in MYO18B is associated with severe nemaline myopathy with cardiomyopathy. Neuromuscular Disord 2015;25:S186-S186.

[14] Prag S, Adams JC. Molecular phylogeny of the kelch-repeat superfamily reveals an expansion of $\mathrm{BTB} / \mathrm{kelch}$ proteins in animals. BMC Bioinformatics $2003 ; 4: 42$.

[15] Gong W, Gohla RM, Bowlin KM, Koyano-Nakagawa N, Garry DJ, Shi X. Kelch Repeat and BTB Domain Containing Protein 5 (Kbtbd5) Regulates Skeletal Muscle Myogenesis through the E2F1-DP1 Complex. J Biol Chem 2015;290:15350-61.

[16] Garg A, O'Rourke J, Long C, et al. KLHL40 deficiency destabilizes thin filament proteins and promotes nemaline myopathy. J Clin Invest 2014;124:3529-39.

[17] Kawase K, Nishino I, Sugimoto M, et al. Nemaline myopathy with KLHL40 mutation presenting as congenital totally locked-in state. Brain Dev 2015;37:887-90. [18] Natera-de Benito D, Nascimento A, Abicht A, et al. KLHL40-related nemaline myopathy with a sustained, positive response to treatment with acetylcholinesterase inhibitors. J Neurol 2016.

[19] Cheng WY, Hakenberg J, Li SD, Chen R. DIVAS: a centralized genetic variant repository representing 150000 individuals from multiple disease cohorts.

Bioinformatics 2016;32:151-3. 
[20] Li MX, Gui HS, Kwan JS, Bao SY, Sham PC. A comprehensive framework for prioritizing variants in exome sequencing studies of Mendelian diseases. Nucleic Acids Res 2012;40:e53.

[21] Van Durme J, Delgado J, Stricher F, Serrano L, Schymkowitz J, Rousseau F. A graphical interface for the FoldX forcefield. Bioinformatics $2011 ; 27: 1711-2$.

[22] Jungbluth $\mathrm{H}$, Sewry CA, Counsell $\mathrm{S}$, et al. Magnetic resonance imaging of muscle in nemaline myopathy. Neuromuscul Disord 2004;14:779-84. 


\section{Mild clinical presentation in KLHL40-related Nemaline Myopathy (NEM 8)}

\section{Figure legends}

Figure 1 Clinical presentation at 8 years: slender phenotype, hypomimia $(A)$, scapular winging $(B)$, facial weakness $(\mathrm{C})$

Figure 2 Muscle biopsy from biceps brachii showing rod clusters (nemaline bodies) in the sub sarcolemmal region on trichrome Gomori $(A)$; scarce rods in the centre of the muscular fibre on trichrome Gomori (B); electronic microscopy confirmed the presence of nemaline bodies in the sub sarcolemmal region (C-D). Western blot performed on skeletal muscle protein extracts by routine methods using the antiKLHL40 (KBTBD5) form Sigma. A 69-53 KDa band corresponding to KLHL40 in protein extracts from an age-matched control was revealed, while no signal was detectable in the patient's biopsy. Myosin heavy chain was used as a loading control (E).

Figure 3 (A) Pedigree of the proband's family and homozygous for the missense mutation $(T / T)$. Unaffected parents and one sibling were both carriers $(C / T)$ and one sibling wasn't bearer of the mutation $(C / C)$; (B, upper part) Structural modelling of the p.Arg500Cys mutation. The crystal structures of the B kelch domain of KLHL40. The fourth Kelch repeat is in blue. The mutation location is represented by a yellow star. (B, lower part) Focus of the Arg500 residu. The Arginine 500 (Arg500) interacts with the Glutamine 528. Hydrogen bonds are represented in yellow doted lines; (C) Sequence chromatographs of KLHL40 fourth exon of the different family genotype. The mutation location is framed. $+/+$ means homozygous for the $\mathrm{C}$ (or reference nucleotide), +/- means heterozygous for the mutation (T) and -/- homozygous for the mutation; (D) Quantitative expression of the KLHL40 transcript extracted from muscle 
biopsy. CB is a control biopsy from a control patient and PB the patient biopsy. In PB, KLHL40 is overexpressed compared to CB KLHL40 expression; (E) Alignment of the corresponding region of KLHL40 containing the index case's mutation. The Arginine 500 is conserved between various species. 


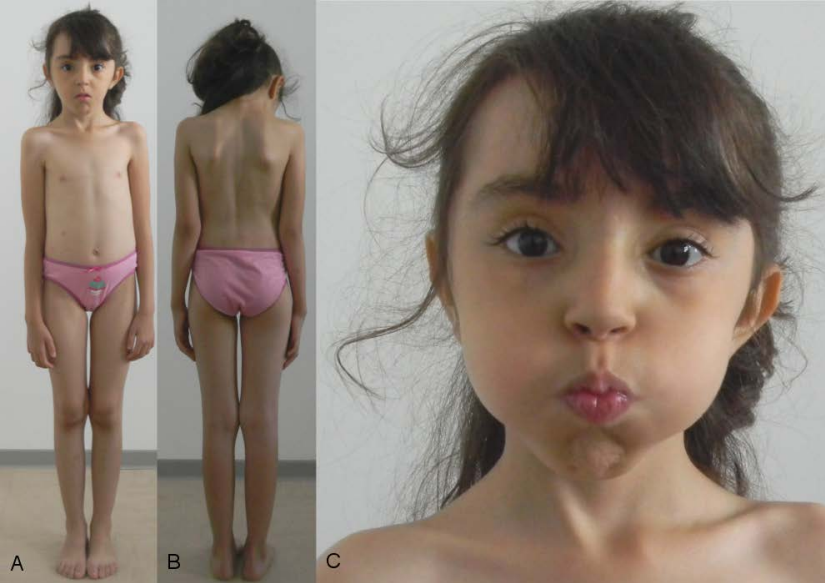



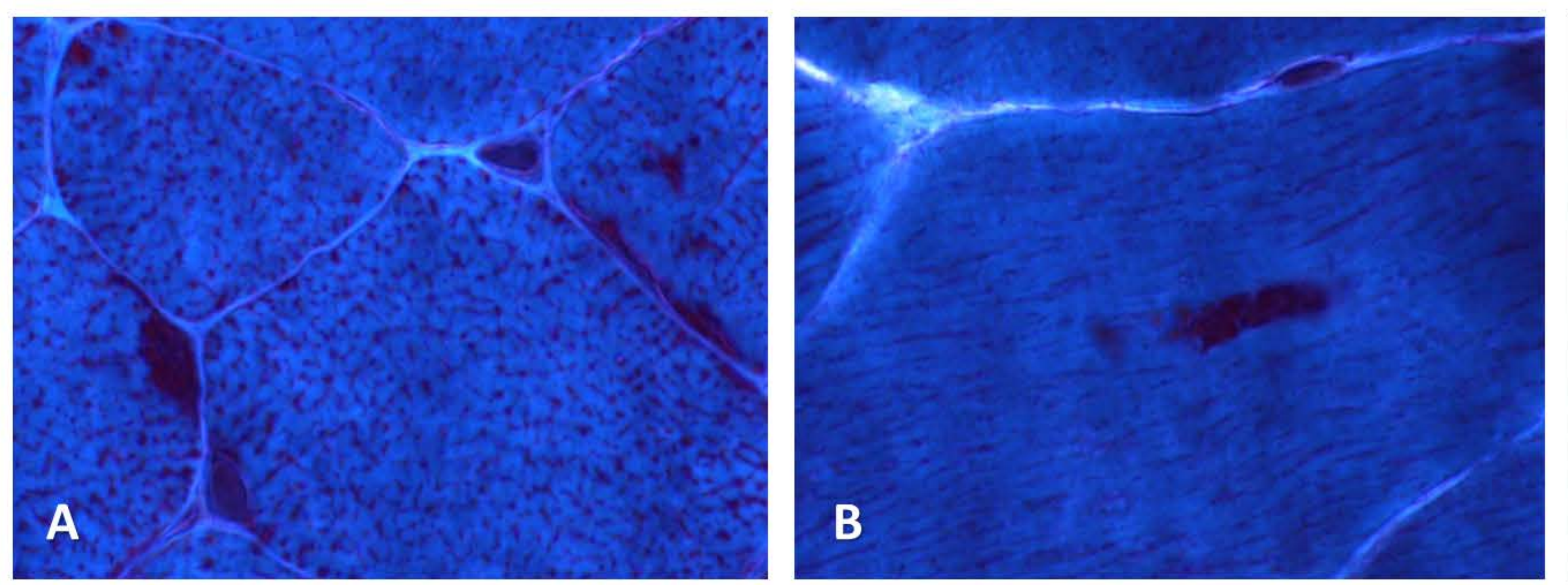

\section{Control}

Patient
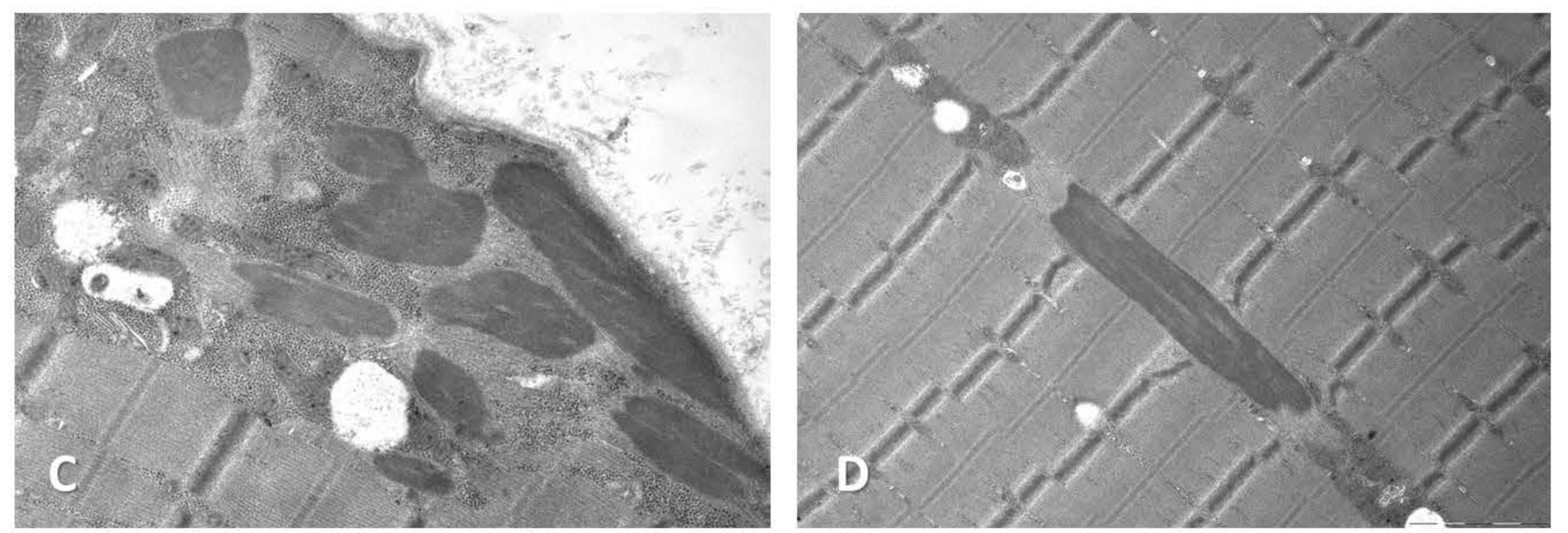

70

50

Myosin

E

KLHL40 
